06

\title{
Модель наноструктурирующего выглаживания сферическим индентором с учетом пластических деформаций
}

\author{
(C) Я.А. Ляшенко, ${ }^{1,2}$ В.Л. Попов ${ }^{1,3,4, \text { Ф }}$ \\ ${ }^{1}$ Берлинский технический университет, \\ 10623 Берлин, Германия \\ ${ }^{2}$ Сумский государственный университет, \\ 40007 Сумы, Украина \\ ${ }^{3}$ Национальный исследовательский Томский государственный университет, \\ 634050 Томск, Россия \\ ${ }^{4}$ Национальный исследовательский Томский политехнический университет, \\ 634050 Томск, Россия \\ ฯ e-mail: v.popov@tu-berlin.de
}

(Поступило в Редакцию 30 мая 2017 г.)

\begin{abstract}
Предложена динамическая модель наноструктурирующего выглаживания поверхности металлических деталей с учетом пластических деформаций. Для описания пластичности используется идеология метода редукции размерности, дополненного критерием пластичности. Модель учитывает действие нормальной выглаживающей силы и тангенциальной силы трения. Получены профили пластического следа после процедуры выглаживания. Исследовано влияние на кинетику выглаживания коэффициента трения, а также периодических осцилляций выглаживающей силы.
\end{abstract}

DOI: 10.21883/JTF.2018.01.45481.2363

\section{Введение}

Одной из актуальных задач современной инженерии является модификация физических свойств поверхности [1]. Для такой модификации применяется много различных методов - это ионная имплантация, лазерная обработка, плазменная обработка, напыление покрытий в вакуумной среде и многое другое. Целью всех этих методов является изменение поверхностных свойств материала таким образом, чтобы они максимально подходили для конкретного инженерного приложения. Это может быть получение поверхностного слоя с повышенной температурой плавления, измененным показателем преломления, уменьшенным износом, повышенной пластичностью (либо твердостью), коррозионной устойчивостью и многое другое. Основная идея модификации поверхности состоит в том, что отпадает необходимость изготавливать всю деталь из дорогостоящего материала, поскольку взаимодействует она с окружающей средой (другими телами) только через поверхность, и здесь важны не объемные, а контактные (поверхностные) свойства.

Одним из эффективных и перспективных методов модификации поверхности является наноструктурирующее выглаживание [2-4]. В процессе выглаживания индентор (как плавило, сферический) погружается на микрометровые глубины в поверхность детали (контролируется глубина индентирования либо действующая нормальная сила), и со скоростью порядка метра в секунду за несколько проходов обрабатывает деталь. При этом в зоне контакта возникают интенсивные пластические деформации, действие которых приводит к фрагментации поверхностного слоя детали и образованию структурных дефектов, в том числе и границ зерен. При этом с каждым проходом индентора средняя величина зерна будет стремиться к конкретному значению, которое зависит от величины пластических деформаций, а также температуры в зоне контакта [5,6]. В итоге у выглаженной детали улучшаются прочностные и пластические характеристики, а также повышается износостойкость. Преимущество метода наноструктурирующего выглаживания в том, что он не требует наличия дорогостоящего оборудования и при этом имеет высокую эффективность. Важным моментом является то, что указанный метод может применяться для выглаживания деталей с отверстиями [7].

Несмотря на то, что рассматриваемый метод наноструктурирующего выглаживания активно используется в промышленности, мы до сих пор далеки от полного понимания процессов, происходящих в процессе выглаживания. Одно из развиваемых теоретических направлений исследования процесса выглаживания основывается на применении компьютерного моделирования. Здесь используется как метод конечных элементов [8], так и метод подвижных клеточных автоматов [9]. Другим направлением является развитие различных феноменологических моделей [7,10]. Конечно феноменологические модели не в состоянии точно описать все процессы, происходящие при выглаживании детали, однако их неоспоримым достоинством является то, что становится возможным установить общие закономерности между результатом процесса и параметрами модели. К примеру, в работе [10] найдено оценочное значение критической величины демпфирования, при превышении которой прекращается режим автоколебаний. Это имеет практическую важность при выглаживании деталей с 
отверстиями, поскольку при выходе индентора из отверстия он приподнимается над поверхностью детали (выходит из контакта), после чего важно, чтобы система за как можно меньшее время вышла на стационарный режим выглаживания, не допустив при этом колебаний индентора, которые приведут к порче поверхности детали [7]. В настоящей работе, основываясь на моделях [7], [10], мы предложили модель наноструктурирующего выглаживания с учетом пластических деформаций. Для описания пластической деформации была использована идеология метода редукции размерности [11], который был дополнен критерием пластичности [12].

\section{Формулировка модели в рамках формализма MDR}

На рис. 1 схематически показан процесс наноструктурирующего выглаживания. Здесь сферический индентор массой $m$ скользит по поверхности детали (показана пружинами) в положительном направлении оси $O X$ с помощью внешнего привода, скорость которого постоянна и равна $V_{0}$. Нормальная сила, под действием которой индентор погружается в субстрат, обозначена как $F_{b}$ (сила выглаживания). Коэффициент демпфирования в нормальном направлении равен $\eta_{z}$. Движущий привод связан с индентором телом Кельвина-Фойгта с упругой силой $F_{x}$ и демпфером $\eta_{x}$. При движении индентора между его поверхностью и поверхностью детали возникает сила трения $F_{f r}$, показанная на рисунке. Запишем уравнение движения индентора в нормальном направлении, оно будет иметь вид [10]

$$
m \frac{\mathrm{d}^{2} z}{\mathrm{~d} t^{2}}+\eta_{z} \frac{\mathrm{d} z}{\mathrm{~d} t}=F_{b}-F_{n},
$$

где введена нормальная контактная сила $F_{n}$, действующая со стороны детали. Для определения эволюции контактной силы в процессе движения будем применять метод редукции размерности (MDR) [11], который позволяет точно описывать контактные задачи аксиально-

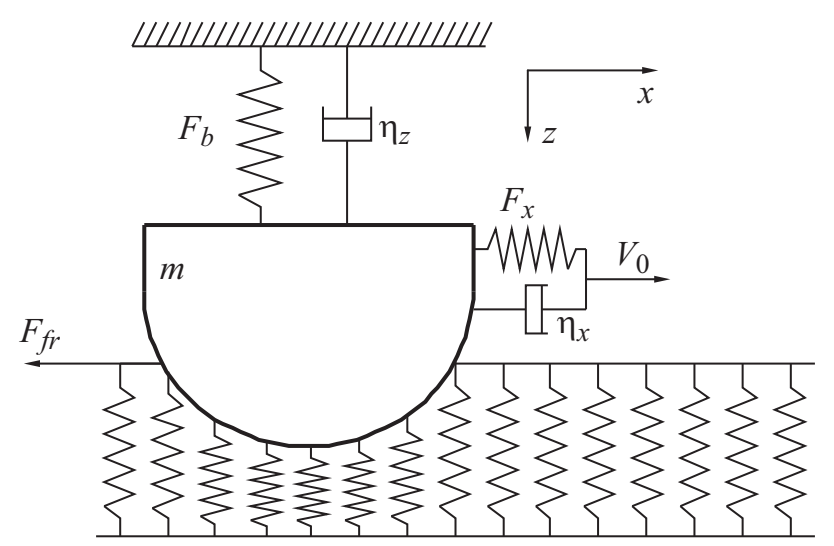

Рис. 1. Схема движения индентора в процессе наноструктурирующего выглаживания. симметричных тел. В рассматриваемом здесь контексте метод не является точным и предлагаемый анализ следует рассматривать как качественный анализ основных закономерностей процесса. Рассмотрим сначала особенности метода MDR без учета пластической деформации. Пусть индентор изготовлен из материала с модулем упругости $E_{1}$, модулем сдвига $G_{1}$ и коэффициентом Пуассона $v_{1}$. Аналогичные параметры для выглаживаемой детали равны $E_{2}, G_{2}$ и $v_{2}$. Известно, что такая контактная задача может быть сведена к задаче контакта несжимаемого индентора с полупространством с эффективными упругими параметрами $E^{*}$ и $G^{*}$, которые определяются как

$$
\begin{gathered}
\frac{1}{E^{*}}=\frac{1-v_{1}^{2}}{E_{1}}+\frac{1-v_{2}^{2}}{E_{2}}, \\
\frac{1}{G^{*}}=\frac{2-v_{1}}{4 G_{1}}+\frac{2-v_{2}}{4 G_{2}} .
\end{gathered}
$$

Далее необходимо дискретизировать полупространство, представив его в виде невзаимодействующих пружин, имеющих нормальную и тангенциальную жесткости,

$$
k_{z}=E^{*} \Delta x, \quad k_{x}=G^{*} \Delta x,
$$

где $\Delta x-$ величина дискретизации (расстояние между пружинами). При вдавливании индентора с оригинальным трехмерным профилем $z=f(r)$ в одномерном пространстве его необходимо представить в виде

$$
g(x)=|x| \int_{0}^{|x|} \frac{f^{\prime}(r)}{\sqrt{x^{2}-r^{2}}} \mathrm{~d} r .
$$

Для сферического индентора $f(r)=r^{2} / 2 R$ преобразование (5) приводит к зависимости $g(x)=x^{2} / R$. В итоге при погружении индентора в полупространство на глубину $d$ натяжения индивидуальных пружин могут быть рассчитаны как

$$
u_{z}(x)=d-g(x)=d-\frac{x^{2}}{R}
$$

Из соотношения (6) легко найти радиус контакта $a$. Так, при $x \equiv a$ и $u_{z}(a)=0$ будем иметь $a=\sqrt{R d}$, что, конечно же, совпадает с классическим решением. Зная глубину индентирования $d$, можно определить действующую нормальную силу $F_{n}$. Для этого необходимо сложить силы натяжения от каждой пружины в контакте:

$$
\begin{aligned}
F_{z}(a) & =E^{*} \int_{-a}^{a} u_{z}(x) \mathrm{d} x=2 E^{*} \int_{0}^{\sqrt{R d}}\left(d-\frac{x^{2}}{R}\right) \mathrm{d} x \\
& =\frac{4 E^{*} \sqrt{R d^{3}}}{3} .
\end{aligned}
$$

Однако при численном моделировании необходимо пользоваться дискретным представлением интеграла, 
т. е. использовать выражение вида

$$
F_{z}=E^{*} \Delta x \sum_{\text {cont }} u_{z}\left(x_{i}\right)
$$

где производится суммирование по всему контакту. Функция $u_{z}\left(x_{i}\right)$, как и ранее, задается выражением (6).

При обработке детали индентор движется в горизонтальном направлении, генерируя силу трения, которая также оказывает влияние на процесс выглаживания. Метод редукции размерности позволяет описать и силу трения. Известно, что в случае, когда материалы, из которых изготовлены индентор и выглаживаемая поверхность, обладают упругим подобием, т.е. когда выполняется условие

$$
\frac{1-2 v_{1}}{G_{1}}=\frac{1-2 v_{2}}{G_{2}},
$$

нормальная и тангенциальная задачи могут рассматриваться независимо.

Опишем кратко процедуру моделирования тангенциального движения индентора в рамках метода MDR с конечным коэффициентом трения $\mu$. Для учета скольжения на каждой итерации для всех пружин проверяется условие

$$
\mu E^{*} u_{z}\left(x_{i}\right) \leqslant\left|G^{*} u_{x}\left(x_{i}\right)\right|,
$$

где $u_{x}\left(x_{i}\right)$ - тангенциальные смещения пружин в контакте. Если условие (10) выполняется, то пружина будет скользить. Для учета скольжения на каждом шаге к горизонтальному смещению пружин инкрементально прибавляется смещение индентора, после чего для всех пружин проверяется условие (10). Для тех пружин, для которых это условие выполняется, устанавливается новая величина смещения

$$
u_{x}\left(x_{i}\right)= \pm \frac{\mu E^{*} u_{z}\left(x_{i}\right)}{G^{*}}
$$

где знак совпадает со знаком $u_{x}\left(x_{i}\right)$ до присваивания величине $u_{x}\left(x_{i}\right)$ нового значения. После этого тангенциальная сила (сила трения) $F_{f r}$ рассчитывается по формуле

$$
F_{f r}=G^{*} \Delta x \sum_{c o n t} u_{x}\left(x_{i}\right)
$$

Описанная методика использовалась ранее для описания влияния на коэффициент трения действия ультразвука [13]. Такой подход позволяет точно описать процесс упругого деформирования. Однако в процессе выглаживания деталь деформируется пластически. Описание нелокальной пластичности не представляется возможным в рамках MDR. Одной из причин этого является то, что при тангенциальном движении и наличии пластической деформации контакт не является аксиально симметричным. Например, в задаче, показанной на рис. 1 (при постоянной глубине индентирования), пружины слева от центра контакта будут максимально пластически деформированы, в то время как пружины в правой части контакта будут продолжать деформироваться. Таким образом, нормальная сила справа будет выше, а это не соответствует идеологии MDR. Поэтому проведенное далее исследование нужно понимать не как точное описание процесса, а как его моделирование, направленное на описание возможных режимов наноструктурирующего выглаживания.

В работе [12] предложен критерий пластической деформации, который мы будем использовать в нашем моделировании. Нормальная сила может быть записана в виде

$$
F_{n}=\pi q_{c} a,
$$

где $a-$ радиус контакта, а $q_{c}$ имеет смысл критической линейной плотности силы, при превышении которой начинается пластическая деформация. Критическая сила $f_{c}$, действующая на одну пружину, может быть найдена как

$$
f_{c}=\frac{F_{n}}{2 a / \Delta x} \text {. }
$$

Из (13) и (14) следует выражение для определения $f_{c}[12]$

$$
f_{c}=\frac{\pi}{2} q_{c} \Delta x,
$$

где $f_{c}$ - критическая (максимальная) упругая сила, при превышении которой начинается пластическое деформирование образца. Рассмотрим процедуру моделирования пластический деформации для отдельной пружины. Пусть ее сжатие составляет $u_{z}\left(x_{i}\right)$. На каждом шаге проверяется условие

$$
u_{z}\left(x_{i}\right) \geqslant \frac{f_{c}}{E^{*} \Delta x} .
$$

Если (16) выполняется, то устанавливается новая величина упругого натяжения пружины $u_{z}^{\prime}\left(x_{i}\right)=f_{c} /\left(E^{*} \Delta x\right)$. Пластическая глубина индентирования при этом составит $d_{p l}=u_{z}\left(x_{i}\right)-u_{z}^{\prime}\left(x_{i}\right)$. При последующих проверках при выполнении условия (16) уже с новым значением $u_{z}^{\prime}\left(x_{i}\right)$ величина $d_{p l}$ будет накапливаться. В итоге после прохода индентора мы получим пластический след, форма которого определяется совокупностью параметров динамической системы, показанной на рис. 1.

\section{Движение индентора с постоянной скоростью}

Для начала рассмотрим упрощенный случай, в котором индентор сдвигается с постоянной скоростью $V_{0}$, что соответствует значению $\eta_{x} \rightarrow \infty$ (рис. 1). Такая ситуация физически соответствует движению индентора в жестких направляющих [10] и часто реализуется в экспериментах. Все же стоит отметить, что горизонтальные колебания индентора будут иметь место даже в случае использования жестких направляющих, однако по сравнению с амплитудой вертикальных колебаний ими можно пренебречь. В таком случае сила трения не 
влияет на получаемые профили выглаженных поверхностей, поскольку горизонтальная координата линейно нарастает со временем. Однако наличие силы трения необходимо непосредственно в эксперименте, поскольку только в этом случае наблюдается деформирование поверхностных слоев, что и является целью выглаживания.

На рис. 2 показаны результаты моделирования при фиксированных параметрах модели. При численном счете выбирались параметры дискретизации $\Delta x=10^{-7} \mathrm{~m}$ и $\Delta t=10^{-7} \mathrm{~s}$, а уравнение (1) решалось с помощью метода Эйлера. Расчет проводился до момента времени $t_{\max }=4 \mathrm{~ms}$ (этому моменту времени соответствуют окончания зависимостей на рис. 2,a). Результаты, показанные на рисунке, соответствуют выглаживанию стальной поверхности $\left(E_{2}=2.08 \cdot 10^{11} \mathrm{~Pa}, v_{2}=0.3\right)$ алмазным индентором $\left(E_{1}=11.472 \cdot 10^{11} \mathrm{~Pa}, v_{1}=0.2\right)$. Из рисунка следует, что со временем система выходит на стационарный режим работы, в котором нормальная контактная сила $F_{n}$ численно равна силе выглаживания $F_{b}$. Причем с увеличением значения критической плотности силы $q_{c}$ уменьшается глубина индентирования. Значение $q_{c}$ является характеристикой материала, поэтому его сложно варьировать. Однако при этом не стоит забывать, что глубину индентирования легко увеличить повышением значения выглаживающей силы $F_{b}$. При этом стоит учитывать, что с ростом нормальной силы будет пропорционально увеличиваться сила трения $F_{f r}$ (поскольку возрастает площадь контакта), поэтому диссипация механической энергии также будет увеличиваться, что может привести к плавлению металла в зоне

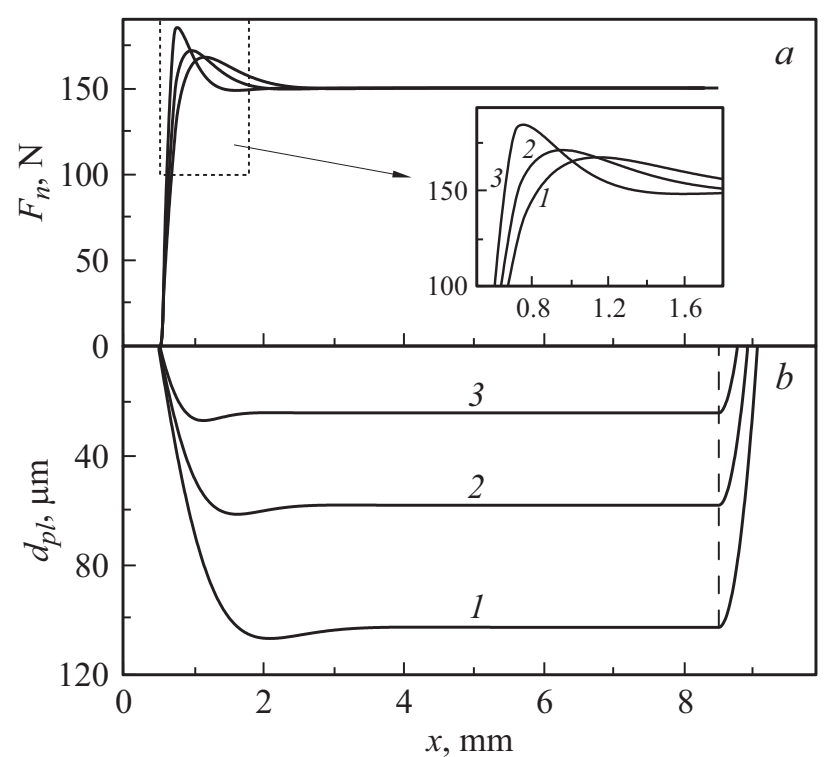

Рис. 2. $a-$ зависимости действующей нормальной силы $F_{n}$ от горизонтальной координаты индентора $x$; $b-$ пластически деформированный профиль стальной детали после однократного прохождения алмазным индентором. Оба рисунка построены при параметрах $R=3 \mathrm{~mm}, F_{b}=150 \mathrm{~N}$, $v_{x}=2 \mathrm{~m} / \mathrm{s}, m=30 \mathrm{~g}, \eta_{z}=30 \mathrm{~kg} / \mathrm{s}$; кривые $1-3$ отвечают значениям $\pi q_{c} / 2=2.5 \cdot 10^{5}, 3.2 \cdot 10^{5}, 4.5 \cdot 10^{5} \mathrm{~N} / \mathrm{m}$.

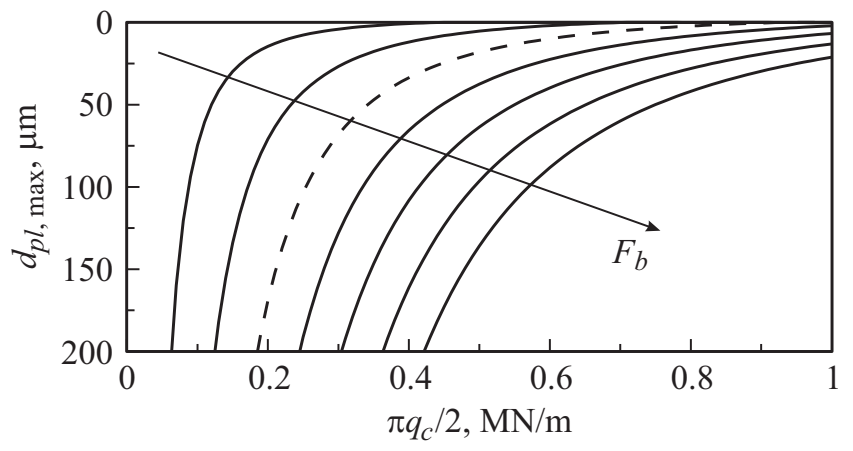

Рис. 3. Зависимости стационарного значения глубины пластического следа $d_{p l, \max }$ от параметра $q_{c}$, построенные при параметрах рис. 2. Значения выглаживающей силы $F_{b}$ увеличиваются от 50 до $350 \mathrm{~N}$ с шагом $50 \mathrm{~N}$ (направление увеличения силы $F_{b}$ на рисунке показано стрелкой). Штриховая кривая соответствует значению $F_{b}=150 \mathrm{~N}$, как и на рис. 2 .

контакта либо образованию трещин. Это в свою очередь накладывает существенные ограничения на величину выглаживающей силы, поэтому влияние всех параметров (нормальная сила, коэффициент трения, масса индентора, и т.д.) следует рассматривать в комплексе, и предложенная модель позволяет это сделать. Кривые на рис. 2 начинаются не из начала координат, поскольку начальная координата центра индентора выбирается равной $x_{0}=0.5 \mathrm{~mm}$. Окончания зависимостей на рис. $2, a$ соответствуют остановке индентора, и далее расчет не ведется. Поэтому начиная с координаты, при которой заканчиваются зависимости на рис. $2, a$, глубина пластического следа $d_{p l}$ на рис. $2, b$ начинает уменьшаться, пока не станет нулевой (поверхность детали, не подверженная обработке). На всех зависимостях $d_{p l}(x)$ перед выходом на стационарный режим выглаживания реализуется минимум (выемка). Такая выемка часто имеет место в экспериментах, что при многократной обработке поверхности детали приводит к автоколебательному режиму выглаживания [10]. Автоколебательный режим может быть описан и в рамках рассматриваемой модели, однако его изучение не является целью настоящей работы, такое исследование было подробно проведено нами в работе [10].

Поскольку основной целью исследования является изучение пластических эффектов, на рис. 3 дополнительно приведены зависимости стационарного значения глубины пластического следа $d_{p l \text {, max }}$ от параметра $q_{c}$, задающего пластические свойства выглаживаемой детали. Из рисунка видно, что для всех кривых с увеличением $q_{c}$ глубина следа уменьшается, пока не становится нулевой (упругое деформирование образца при отсутствии пластической деформации).

\section{Влияние силы трения}

Как мы уже отмечали выше, движение индентора с постоянной скоростью является идеализацией процесса выглаживания. Поэтому рассмотрим случай, в котором 


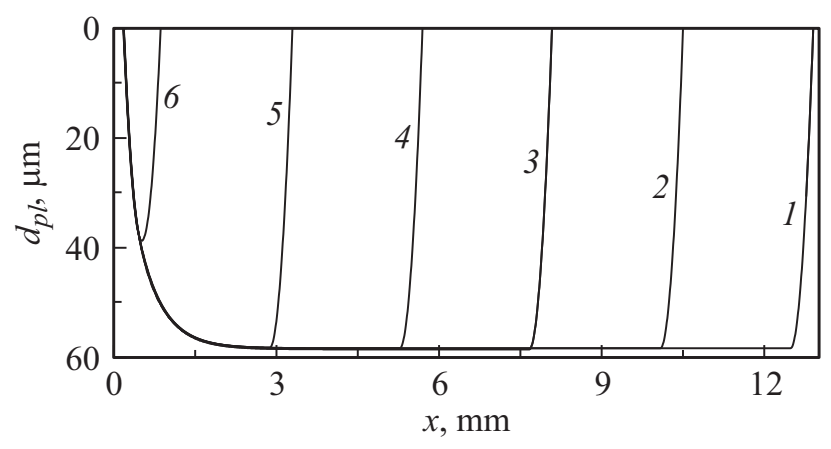

Рис. 4. Пластически деформированный профиль стальной детали после процедуры выглаживания при параметрах рис. 2 и $\pi q_{c} / 2=3.2 \cdot 10^{5} \mathrm{~N} / \mathrm{m}, G_{1}=4.78 \cdot 10^{11} \mathrm{~Pa}$, $G_{2}=8 \cdot 10^{10} \mathrm{~Pa}, \eta_{x}=30 \mathrm{~kg} / \mathrm{s}, F_{x}=60 \mathrm{~N}$. Кривые $1-6$ соответствуют $\mu=0.3,0.32,0.34,0.36,0.38,0.4$.

фиксируется движущая сила $\left(F_{x}=\right.$ const), а не скорость. В такой ситуации существенное влияние на процесс выглаживания оказывает сила трения $F_{f r}$, поскольку она будет изменяться при движении индентора в нормальном направлении. При этом для описания динамики процесса необходимо уравнение для нормальной координаты $z$ (1) дополнить аналогичным уравнением, описывающим движение индентора в горизонтальном направлении. Такое уравнение с учетом действующих сил примет вид

$$
m \frac{\mathrm{d}^{2} x}{\mathrm{~d} t^{2}}+\eta_{x} \frac{\mathrm{d} x}{\mathrm{~d} t}=F_{x}-F_{f r}
$$

Стоит отметить, что кинетические уравнения (1) и (17) не являются независимыми, поскольку сила трения $F_{f r}$ (уравнение (17)) представляет функцию нормальной силы $F_{n}$ (уравнение $\left.(1)\right)$. Результаты соответствующего моделирования показаны на рис. 4 (длительность процесса выглаживания для всех кривых здесь одинакова и составляет $t_{\max }=25 \mathrm{~ms}$ ). Параметры $G_{1}$ и $G_{2}$, используемые при моделировании влияния силы трения, рассчитывались по известному соотношению

$$
G_{1,2}=\frac{E_{1,2}}{2\left(1+v_{1,2}\right)}
$$

Кривые 1-5 на рис. 4 показывают, что с увеличением коэффициента трения $\mu$ за один и тот же промежуток времени при фиксированной движущей силе $F_{x}$ происходит меньшее смещение индентора в тангенциальном направлении. Кривая 6 построена при значении коэффициента трения $\mu=0.4$, при котором отсутствует проскальзывание и реализуется статические трение. Поэтому здесь скольжение (тангенциальное движение) индентора отсутствует, и пластическая деформация наблюдается только в зоне первоначального контакта. С дальнейшим увеличением коэффициента трения зависимости $d_{p l}(x)$ будут повторять вид кривой 6 . Отметим, что на самом деле коэффициент трения в паре алмаз-сталь составляет порядка 0.1, однако мы проводим расчет для бо́льших значений коэффициента трения. Это связано с тем, что индентор может быть изготовлен из различных материалов, а величина коэффициента трения в процессе выглаживания имеет определяющую роль. Если коэффициент трения меньше критического значения, то поверхностный слой детали не будет пластически деформироваться, поэтому обеспечение высокого уровня трения является одним из важных моментов в технологическом процессе наноструктурирующего выглаживания.

\section{Влияние периодических колебаний нормальной силы}

Очень часто для обеспечения необходимого режима работы к индентору прикладывают внешнее периодическое воздействие. Такое воздействие, к примеру, может снижать силу трения [12] и даже создавать направленное движение трущихся поверхностей [14]. В рассматриваемом нами случае внешнее периодическое воздействие может быть описано с помощью осцилляций выглаживающей силы $F_{b}$ в виде

$$
F_{b}=\tilde{F}_{b}+\tilde{F} \cos (2 \pi \tilde{v} t) .
$$

На рис. 5 показаны результаты моделирования при такой зависимости нормальной силы. Значение $\tilde{F}_{b}$ выбрано равным значению выглаживающей силы, при которой были построены предыдущие рисунки. Таким образом, среднее значение силы $\left\langle F_{b}\right\rangle$, действующей на индентор со стороны выглаживающего устройства, не изменяется. Из рисунка следует, что с ростом частоты уменьшается амплитуда волнистости выглаженной детали. В пределе $\tilde{v} \rightarrow \infty$ выглаженная поверхность будет гладкой, как и на предыдущем рисунке.

На рис. 6 показаны зависимости амплитуды колебаний пластического следа $d_{p l}(x)$, показанного на рис. 5, в стационарном режиме выглаживания. Все параметры на рис. 5,6 аналогичны, изменяется только значение коэффициента демпфирования $\eta_{z}$, увеличение которого обозначено стрелкой. Из рисунка следует, что с увеличением частоты $\tilde{v}$ амплитуда волнистости $A_{p l}$ уменьшается. Уменьшается она также и с ростом величины

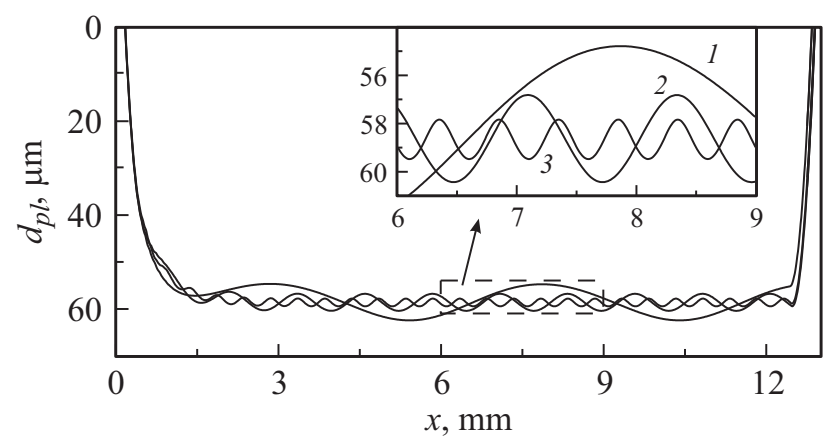

Рис. 5. Пластически деформированный профиль стальной детали при параметрах рис. $4, \mu=0.3, \tilde{F}_{b}=150 \mathrm{~N}$ и $\tilde{F}=5 \mathrm{~N}$. Кривые $1-3$ соответствуют частотам $\tilde{v}=100,400,1000 \mathrm{~Hz}$. 


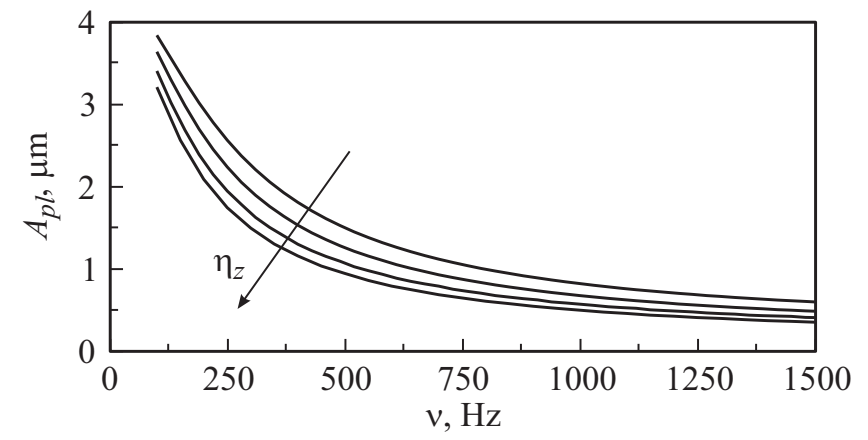

Рис. 6. Амплитуда колебаний пластического следа $A_{p l}$, показанного на рис. 5 , как функция частоты изменения нормальной силы $\tilde{v}$. Кривые, показанные на рисунке, соответствуют значениям $\eta_{z}=30,250,500,700 \mathrm{~kg} / \mathrm{s}$.

демпфирования $\eta_{z}$, однако несущественно. В работе [10] показано, что с превышением коэффициентом демпфирования критической величины режим автоколебаний исчезает. Однако разница в том, что в [10] рассматривалась ситуация, когда нормальная сила оставалась фиксированной, и колебания осуществлялись за счет наличия в детали отверстий. Здесь же рассматриваются внешние колебания нормальной силы. И, как видно, значительное увеличение коэффициента демпфирования снижает амплитуду колебаний несущественно.

Стоит отметить, что подобное периодическое изменение величины нормальной силы в процессе выглаживания может быть вызвано наличием отверстий в детали, расположенных в ряд по ходу индентора. И, как видно, действие периодического воздействия в широком диапазоне параметров приводит к волнистости выглаженной детали. Одной из принципиальных возможностей избежать такого эффекта является построение интеллектуальных систем, которые будут регулировать подачу индентора при изменении нормальной выглаживающей силы, тем самым задавая ее постоянное значение. В таком случае становится возможным выглаживание поверхностей с отверстиями либо неровностями.

Стоит сказать, что описанный режим выглаживания с „волнистостью“ может иметь и положительный эффект. В случае двигателя внутреннего сгорания либо гидравлических систем такой режим может использоваться для нанесения на поверхность поршня канавок микрометровой глубины, в которых будет находиться смазочный материал при его движении в цилиндре.

\section{Заключение}

В настоящей работе предложена простая модель, позволяющая проводить численное моделирование динамического процесса наноструктурирующего выглаживания. При этом как функции времени могут быть определены радиус контакта, нормальная и тангенциальная контактные силы, пластический след после выглаживания, а также положение индентора в пространстве и скорость его движения. С помощью модели проанализировано влияние силы трения на динамику процесса и показано, что увеличение коэффициента трения приводит к уменьшению скорости движения индентора при фиксированной тангенциальной движущей упругой силе. Изучение влияния наложенных извне осцилляций индентора в нормальном направлении показало, что с ростом частоты колебаний выглаженная поверхность приближается к гладкой. Предложенная нами модель может быть использована для дальнейшего изучения процесса выглаживания, а именно - для изучения влияния осцилляций в тангенциальном направлении, определения влияния формы индентора (используемый метод позволяет проводить моделирование для любой аксиально симметричной формы индентора). Также модель легко обобщить на выглаживание вязко-упругих деталей, на градиентные среды, может быть проведен учет адгезии между контактирующими поверхностями.

Работа выполнена при финансовой поддержке Немецкого научно-исследовательского сообщества (DFG), Министерства образования Российской Федерации, и программы повышения конкурентоспособности Томского государственного университета. Я.А. Ляшенко также выражает благодарность МОНУ за частичную финансовую поддержку в рамках проекта № 0116U006818.

\section{Список литературы}

[1] Погребняк А.Д., Братушка С.Н., Береснев В.М., Levintant-Zayonts N. // Успехи химии. 2013. Т. 82. № 12. C. $1135-1159$.

[2] Hassan A.M., Al-Bsharat A.S. // Wear. 1996. Vol. 199. P. 1-8.

[3] Korzynski M. // Int. J. Mach. Tools Manuf. 2007. Vol. 47. P. 1956-1964.

[4] Swirad S. // Wear. 2011. Vol. 271. P. 576-581.

[5] Хоменко А.В., Ляшенко Я.А., Метлов Л.С. // МФиНТ. 2008. T. 30. № 6. C. 859-872.

[6] Метлов Л.С., Мышляев М.М., Хоменко А.В., Ляшенко Я.А. // Письма в ЖТФ. 2012. Т. 38. Вып. 21. С. 28-33.

[7] Кузнецов В.П., Ильичев С.А., Горгоч, В.Г. // Вестник машиностроения. 2009. № 11. С. 71-75.

[8] Кузнецов В.П., Смолин И.Ю., Дмитриев А.И., Коновалов Д.А., Макаров А.В., Киряков А.Е., Юровских А.С. // Физ. мезомех. 2013. Т. 14. № 6. С. 87-97.

[9] Kuznetsov V.P., Tarasov S.Yu., Dmitriev A.I. // J. Mater. Process. Technol. 2015. Vol. 217. P. 327-335.

[10] Ляменко Я.А., Кузнецов В.П., Попов М., Попов В.Л., Горгои В.Г. // Физ. мезомех. 2015. Т. 18. № 1. С. 38-42.

[11] Popov V.L., Heß M. Method of dimensionality reduction in contact mechanics and friction. Berlin: Springer, 2015. $265 \mathrm{p}$.

[12] Popov V.L. // FU Mech. Eng. 2015. Vol. 13. N 1. P. 39-46.

[13] Teidelt E., Popov V.L., Starcevic J. // Tribol. Lett. 2012. Vol. 48. P. 51-62.

[14] Cheng T.H., He M., Li H.Y., Lu X.H., Zhao H.W., Gao H.B. // IEEE Trans. Ind. Electron. 2017. Vol. 64. N 7. P. 5545-5552. 